\title{
Pentalenolactone Biosynthesis. Molecular Cloning and Assignment of Biochemical Function to PtIl, a Cytochrome P450 of Streptomyces avermitilis
}

\author{
Richard Quaderera , Satoshi Omura ${ }^{\mathrm{b}}$, Haruo Ikeda ${ }^{\mathrm{C}}$, and David E. Cane ${ }^{\mathrm{a},{ }^{*}}$ \\ a Department of Chemistry, Brown University, Box H, Providence, RI 02912-9108, USA \\ b The Kitasato Institute, 9-1, Shirokane 5-chome, Minatoku, Tokyo 108-8642, Japan \\ c Kitasato Institute for Life Sciences, Kitasato University, 1-15-1, Kitasato, Sagamihara, Kanagawa \\ 228-8555, Japan
}

Abstract

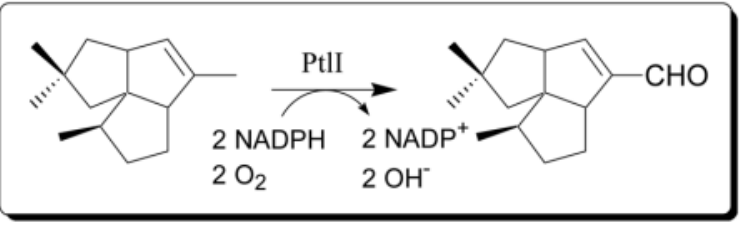

A gene cluster encoding all of the enzymes for the biosynthesis of the antibiotic pentalenolactone (1) has recently been identified inStreptomyces avermitilis. The biosynthetic gene cluster contains theptlI (SAV2999) gene which encodes a cytochrome P450 (CYP183A1). PtlI was cloned by PCR and expressed inEscherichia coli as a C-terminal-His $6^{-}$tag protein. Recombinant PtlI bound pentalenene $(3)$ with high affinity $\left(K_{\mathrm{D}} 1.44 \pm 0.14 \mu \mathrm{M}\right)$. Incubation of recombinant PtII with $( \pm)-3$ in the presence of NADPH,E. coliflavodoxin and flavodoxin reductase, and $\mathrm{O}_{2}$ resulted in conversion to a single enantiomer of pentalen-13-al (7), by stepwise allylic oxidation via pentalen-13-ol (6). The steady state kinetic parameters for the oxidation of pentalenene $(3)$ to pentalen-13-ol $(\mathbf{6})$ were $k_{\text {cat }}$ $0.503 \pm 0.006 \mathrm{~min}^{-1}$ and $K_{\mathrm{m}} 3.33 \pm 0.62 \mu \mathrm{M}$ for3.

Streptomyces are a rich source of bioactive secondary metabolites. The $9.03 \mathrm{Mb}$ linear chromosome of $S$. avermitilis, the producer of the widely used antiparasitic avermectins, harbors 7,575 open reading frames (ORFs) ${ }^{1}$ of which 33 encode cytochrome $\mathrm{P} 450$ enzymes. 2 One of these CYP genes, ptlI (SAV2999, CYP183A1) is found within the gene cluster for the biosynthesis of the sesquiterpene antibiotic pentalenolactone (1). This cluster lies in a 13.4$\mathrm{kb}$ segment, centered at $3.75 \mathrm{Mb}$ in the $S$. avermitilis genome, that contains 13 unidirectionallytranscribed ORFs (Figure 1). ${ }^{3}$ Among these ORFs, the 1011-bp ptlA, encodes pentalenene synthase (PtlA), which catalyzes the cyclization of farnesyl diphosphate (FPP) (2) to pentalenene (3), the established parent hydrocarbon of the pentalenolactone family of antibiotics (Scheme 1). ${ }^{3,4}$ Besides the heme-dependent monooxygenase CYP183A1 (ptll), ${ }^{2}$ seven of the remaining ORFs correspond to redox enzymes, including the non-heme iron dioxygenase encoded by $p t l H,{ }^{5}$ and six additional monooxygenases and dioxygenases.

We recently showed that $\mathrm{PtlH}$, an $\mathrm{Fe}^{2+} / \alpha$-ketoglutarate-dependent hydroxylase, catalyzes the conversion of 1-deoxypentalenic acid (4) to a new biosynthetic intermediate, 11 $\beta$-hydroxy-1- 
deoxypentalenic acid (5). ${ }^{5}$ Although several presumptive intermediates of pentalenolactone biosynthesis have been isolated from a wide variety of Streptomyces species, ${ }^{3,4} \mathrm{PtlH}$ is the only enzyme linking pentalenene (3) to pentalenolactone (1) that has been characterized to date. Here we describe the biochemical characterization of PtII, the cytochrome P450 that is shown to catalyze the conversion of pentalenene (3) to pentalen-13-al (7) by stepwise oxidation via pentalen-13-ol (6).

According to the proposed biosynthetic pathway (Scheme 1), the enzymes responsible for the conversion of pentalenene (3) to pentalenolactone (1) must first oxidize pentalenene to the corresponding unsaturated carboxylic acid 4. Cytochrome P450s are known to catalyze numerous oxygenation reactions of nonactivated hydrocarbons. ${ }^{6}$ Amongst these reactions is the three-step oxidation of a methyl group to a carboxylic acid. ${ }^{7-9} \mathrm{We}$ therefore speculated that PtII might be responsible for all or part of the allylic oxidation of pentalenene (3) to 1deoxypentalenic acid (4) (Scheme 2).

PtlI was amplified by polymerase chain reaction (PCR) from DNA of S. avermitilis cosmid CL_216_D07 and cloned between the NdeI and XhoI sites of the vector pET31b. The resulting construct pET31b-PtlI was transformed into Escherichia coli BL21(DE3). After induction with IPTG, the expressed PtlI protein, carrying a C-terminal His $_{6}$-tag, was purified to homogeneity by Ni-NTA chromatography. ${ }^{10}$ MALDI-TOF MS of purified protein showed subunit $M_{\mathrm{D}} \mathrm{m} /$ $z 51667 \pm 50$ (calc. 51723 for apo-protein) and $m / z 52078 \pm 50$ (calc. 52339 for holo-protein). Treatment of the sodium dithionite-reduced protein with carbon monoxide gave the characteristic P450 UV difference spectrum. ${ }^{11}$

Titration of PtII with pentalenene (3) ${ }^{12}$ resulted in the typical blue-shift from $420 \mathrm{~nm}$ to 390 $\mathrm{nm}$ (type I binding). ${ }^{13}$ The dissociation constant for 3 was determined by non-linear fitting of the UV-difference spectra to give $K_{\mathrm{D}}=1.44 \pm 0.14 \mu \mathrm{M}$. By contrast, the control sesquiterpene (-)-trans-caryophyllene showed no type I binding when added to PtlI.

A mixture of recombinant PtlI $(0.57 \mu \mathrm{M})$, E. coli flavodoxin (Fld, $3.9 \mu \mathrm{M}),{ }^{14,15 \mathrm{a}}$ E. coli flavodoxin reductase $(\mathrm{Fdr}, 6.3 \mu \mathrm{M}),{ }^{14,15 \mathrm{~b}} \mathrm{NADPH}(0.45 \mathrm{mM})$, and a NADPH-regeneration system [glucose-6-phosphate $(3.1 \mathrm{mM})$ and glucose-6-phosphate dehydrogenase $(10 \mathrm{u})$ ] in 3.0 $\mathrm{mL}$ of $50 \mathrm{mM}$ phosphate buffer, $10 \%$ glycerol (v/v), $\mathrm{pH} 7.4$, was incubated with ( \pm )-(3) (1.1 $\mathrm{mM}$ ) plus $0.8 \%$ DMSO for $16 \mathrm{~h}$ at room temperature. GC-MS analysis of the pentane extract revealed exclusively two new peaks with $\mathrm{m} / 2.218$ (retention time $10.96 \mathrm{~min}$ ) and 220 (retention time $11.03 \mathrm{~min}$ ), identical to authentic pentalen-13-al (7) and pentalen-13-ol (6), respectively (Figure 2 and Supporting Information). ${ }^{12}$ The ${ }^{1} \mathrm{H}$ NMR spectrum of the crude neutral extract also showed the characteristic aldehydic and olefinic signals at $\delta 9.71$ and $6.704(\mathrm{~d}, J=0.8 \mathrm{~Hz})$, respectively for 7 (Figure S8). Chiral GC-MS analysis, under conditions in which individual enantiomers of ( \pm )-pentalen-13-ol (6) and ( \pm )-pentalen-13-al (7) were well resolved, confirmed that enzymatically-produced $\mathbf{6}$ and $\mathbf{7}$ were each single enantiomers. Preparative-scale incubation with ( \pm )-pentalenene (3) gave a mixture containing 6 and 7 , which was dissolved in methanol and treated with sodium borohydride to give alcohol $\mathbf{6}$, identical by ${ }^{1} \mathrm{H}$ NMR to chemically synthesized pentalen-13-ol (6). Incubation using alcohol $\mathbf{6}$ as substrate confirmed that PtII catalyzes the oxidation of 6 to aldehyde $7 .{ }^{16}$ By contrast, only trace amounts of 1deoxypentalenic acid $\mathbf{4}$ could be detected under a wide variety of incubation conditions.

PtlI showed a pH optimum of 8.0 for the oxidation of pentalenene to pentalen-13-ol. The apparent steady-state kinetic parameters for the first oxidation step were determined by carrying out a series of 10-min incubations with 4-40 $\mu \mathrm{M}$ of ( \pm )-pentalenene (3) and quantitation of the product pentalen-13-ol (6) by GC-MS. Under these conditions, further oxidation of 6 was negligible. ${ }^{8 b}$ Fitting of the initial velocities to the Michaelis-Menten 
equation gave $k_{\text {cat }} 0.503 \pm 0.006 \mathrm{~min}^{-1}$ and a $K_{\mathrm{m}}$ of $3.33 \pm 0.62 \mu \mathrm{M}$ for the active enantiomer of 3.

These results establish that the $p t l I$ gene product can catalyze the two-step oxidation of pentalenene (3) to pentalen-13-al (7) (Scheme 2). At this point, it remains an open question how aldehyde 7 gets converted to 1-deoxypentalenic acid (4). Although it remains possible that PtII might support the latter oxidation under the appropriate conditions, ${ }^{17}$ by analogy to other P450s, ${ }^{7-9}$ it is also conceivable that another redox enzyme from within the biosynthetic gene cluster could be responsible for this conversion. The work reported here sheds new light on the biosynthetic gap between pentalenene (3), generated by PtlA-catalyzed cyclization of FPP, and 11 $\beta$-hydroxy-1-deoxypentalenic acid (5), the product of PtlH-catalyzed hydroxylation of 1-deoxypentalenic acid. Biochemical characterization of the remaining ORFs of the pentalenolactone biosynthetic gene cluster is in progress.

\section{Supplementary Material}

Refer to Web version on PubMed Central for supplementary material.

\section{Acknowledgements}

We thank Dr. Tun-Li Shen for determining the mass spectra and Dr. Michael Waterman, Vanderbilt University, for providing us with plasmids harboring fld/fdr. Plasmid pPtlI-camAB encoding putidaredoxin and putidaredoxin reductase was generously provided by Mercian Corp, Japan. This work was supported by NIH grant GM30301 to DEC, by Grant of the 21st Century COE Program, Ministry of Education, Culture, Sports, Science and Technology, Japan to H.I and S.O, by Grant-in-Aid for Scientific Research of the Japan Society for the Promotion of Science No. 17510168 to H.I and by a postdoctoral fellowship by the Swiss National Funds to R.Q.

\section{References and Notes}

1. (a) Omura S, Ikeda H, Ishikawa J, Hanamoto A, Takahashi C, Shinose M, Takahashi Y, Horikawa H, Nakazawa H, Osonoe T, Kikuchi H, Shiba T, Sakaki Y, Hattori M. Proc Natl Acad Sci U S A 2001;98:12215-12220. [PubMed: 11572948] (b) Ikeda H, Ishikawa J, Hanamoto A, Shinose M, Kikuchi H, Shiba T, Sakaki Y, Hattori M, Omura S. Nature Biotech 2003;21:526-531.

2. Lamb DC, Ikeda H, Nelson DR, Ishikawa J, Skaug T, Jackson C, Omura S, Waterman MR, Kelly SL. Biochem Biophys Res Commun 2003;307:610-619. [PubMed: 12893267]

3. Tetzlaff CN, You Z, Cane DE, Takamatsu S, Omura S, Ikeda H. Biochemistry 2006;45:6179-6186. [PubMed: 16681390]

4. (a) Cane DE, Sohng JK, Lamberson CR, Rudnicki SM, Wu Z, Lloyd MD, Oliver JS, Hubbard BR. Biochemistry 1994;33:5846-5857. [PubMed: 8180213] (b) Cane DE, Oliver JS, Harrison PHM, Abell C, Hubbard BR, Kane CT, Lattman R. J Am Chem Soc 1990;112:4513-4524.

5. (a) You Z, Omura S, Ikeda H, Cane DE. J Am Chem Soc 2006;128:6566-6567. [PubMed: 16704250] (b) The 1-deoxypentalenic acid (4) has been isolated as the glucuronate ester: Takahashi S, Takeuchi M, Arai M, Seto H, Otake N. J Antibiot 1983;36:226-228. [PubMed: 6833143]

6. Sono M, Roach MP, Coulter ED, Dawson JH. Chem Rev 1996;96:2841-2887. [PubMed: 11848843]

7. Gibberellin biosynthesis: (a) Helliwell CA, Poole A, Peacock WJ, Dennis ES. Plant Physiol 1999;119:507-510. [PubMed: 9952446] (b) Helliwell CA, Chandler PM, Poole A, Dennis ES, Peacock WJ. Proc Natl Acad Sci U S A 2001;98:2065-2070. [PubMed: 11172076]

8. Bile acid biosynthesis: (a) Cali JJ, Russell DW. J Biol Chem 1991;266:7774-7778. [PubMed: 1708392] (b) Pikuleva IA, Babiker A, Waterman MR, Bjorkhem I. J Biol Chem 1998;273:18153-18160. [PubMed: 9660774]

9. Artemisinic acid biosynthesis: (a) Ro DK, Paradise EM, Ouellet M, Fisher KJ, Newman KL, Ndungu JM, Ho KA, Eachus RA, Ham TS, Kirby J, Chang MCY, Withers ST, Shiba Y, Sarpong R, Keasling JD. Nature 2006;440:940-943. [PubMed: 16612385] (b) Teoh KH, Polichuk DR, Reed DW, Nowak G, Covello PS. FEBS Lett 2006;580:1411-1416. [PubMed: 16458889] 
10. The nonionic detergent Triton X-100 (0.1\%) was a required component of the lysis buffer in order for PtlI to bind to the Ni-NTA resin, suggesting that PtlI may be membrane-associated.

11. Omura T, Sato R. J Biol Chem 1964;239:2370-2378. [PubMed: 14209971]

12. Compounds ( \pm )-(3), ( \pm )-(6), ( \pm )-(7), and ( \pm )-(4) were synthesized as previously described: (a) Ohfune Y, Shirahama H, Matsumoto T. Tetrahedron Lett 1976;17:2869-2872. (b) Ohtsuka T, Shirahama H, Matsumoto T. Tetrahedron Lett 1983;24:3851-3854. (c) Cane DE, Tillman AM. J Am Chem Soc 1983;105:122-124.(d)TillmanAMPhD ThesisBrown UnivProvidence, RI1984115163

13. Jefcoate CR. Methods Enzymol 1978;52:258-279. [PubMed: 209288]

14. The natural electron transport pair for PtlI is unknown. S. avermitilis harbors six putative ferredoxin reductases and nine ferredoxins (cf. Ref 2). Numerous attempts to observe PtlI-catalyzed oxidation of pentalenene (3) using typical redox pairs such as spinach ferredoxin and NADPH:ferredoxin oxidoreductase or putidaredoxin and putidaredoxin reductase were uniformly unsuccessful. By contrast, incubations of 3 and NADPH with unpurified cell-free extracts of E. coli PtlI expression cultures indicated that the endogenous E. coli flavodoxin (Fld) and flavodoxin reductase (Fdr) supported activity of PtlI. Although S. avermitilis does not harbor a native flavodoxin, similar observations have been previously reported for other P450s. (a) Jenkins CM, Waterman MR. J Biol Chem 1994;269:27401-27408. [PubMed: 7961651] (b) Zhao B, et al. J Biol Chem 2005;280:11599_ 11607. [PubMed: 15659395]

15. (a) FldA, Swissprot P61949: Jenkins CM, Pikuleva I, Kagawa N, Waterman MR. Arch Biochem Biophys 1997;347:93-102. [PubMed: 9344469](b) Fdr, Swissprot P28861: Jenkins CM, Waterman MR. Biochemistry 1998;37:6106-6113. [PubMed: 9558349]

16. Some background oxidation of ( \pm )-(6) to pentalen-13-al (7) could also be detected. Under typical incubation conditions, the ratio of P450-catalyzed oxidation to oxidation in the absence of PtlI was $\sim 3: 1$, as judged by GC-MS (PtII $(1.4 \mu \mathrm{M})$, Fld $(6.0 \mu \mathrm{M}) \mathrm{Fdr},(3.4 \mu \mathrm{M})$, NADPH $(0.53 \mathrm{mM})$, glucose-6phosphate $(0.53 \mathrm{mM})$ and glucose-6-phosphate dehydrogenase $(5 \mathrm{u})$ in $2.9 \mathrm{~mL}$ of $50 \mathrm{mM}$ Tris-HCl buffer, $10 \%$ glycerol (v/v), $\mathrm{pH} 8$, was incubated with $( \pm)-(6)(0.1 \mathrm{mM})$ for $2 \mathrm{~h}$ at $\left.25^{\circ} \mathrm{C}\right)$. This finding was consistent with chiral GC-MS analysis of an incubation of ( \pm -)(6) with PtII, which revealed formation of both enantiomers of aldehyde 7 in a ratio of 2.6:1 natural 7 to enantio-7.

17. Incubations carried out with PtII, ferredoxin FdxD (SAV3129) and ferredoxin reductase FprD (SAV5675), the most abundant electron carriers in S. avermitilis, showed no enhancement in pentalenene oxidation and no detectable formation of 1-deoxypentalenic acid (4). 


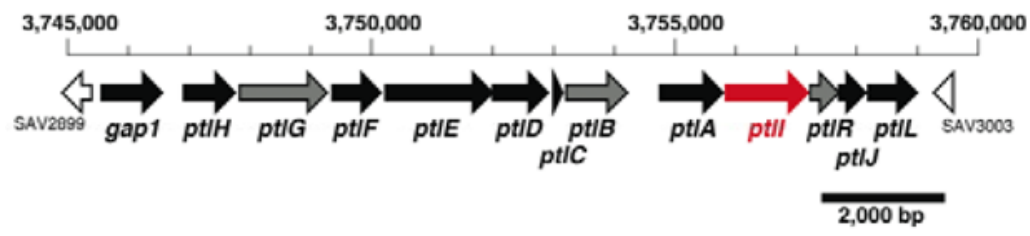

Figure 1.

Pentalenolactone biosynthetic gene cluster from $S$. avermitilis. (See http://avermitilis.ls.kitasato-u.ac.jp/.) 


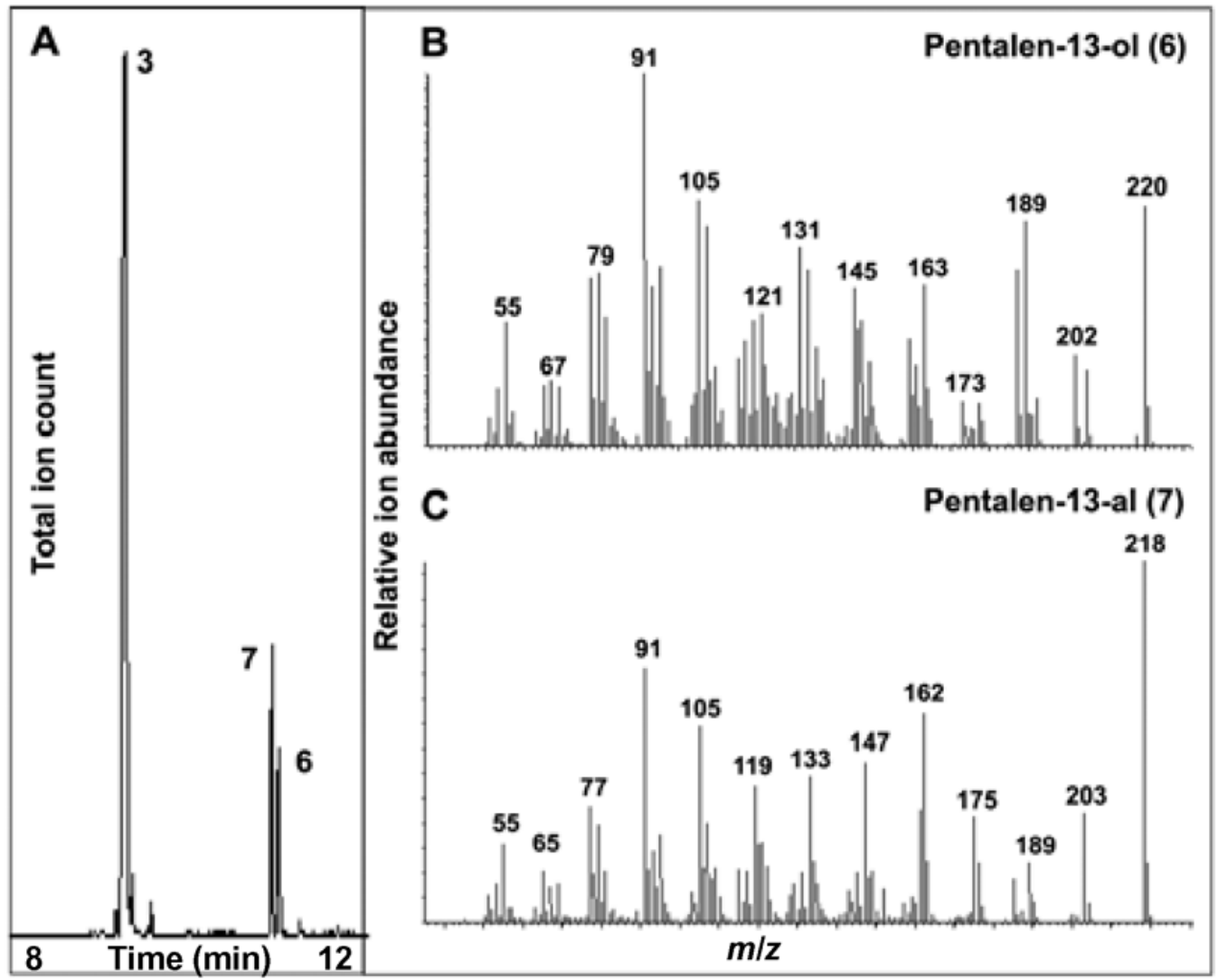

Figure 2.

GC-MS analysis of incubation of PtlI with ( \pm )-pentalenene (3). A, GC trace of pentane extract. B MS of 6 from PtlI-catalyzed oxidation of 3. C MS of 7 from PtlI-catalyzed oxidation of 3. 


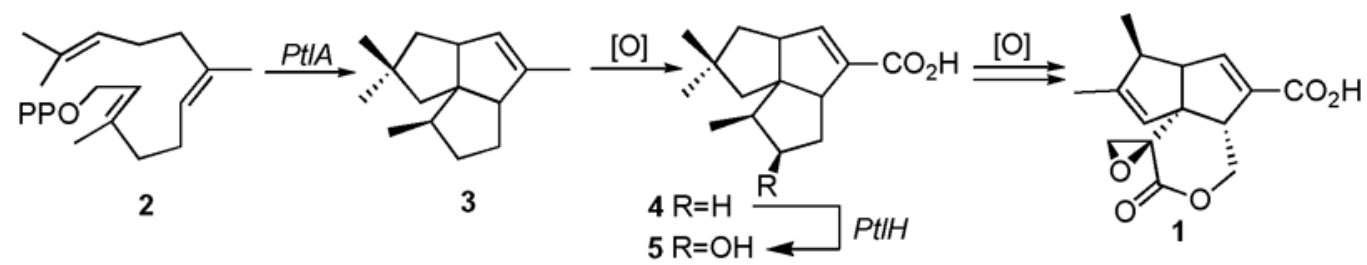

Scheme 1. 


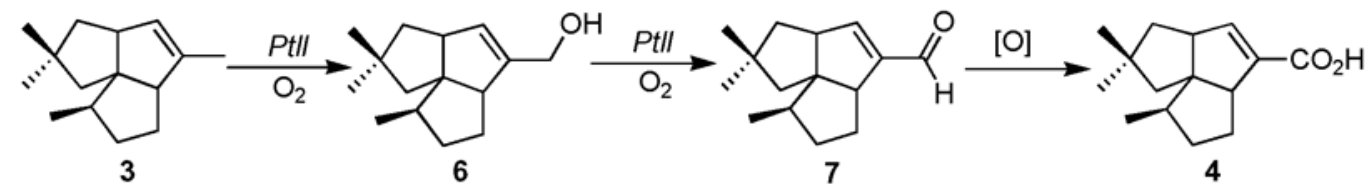

Scheme 2. 